\title{
Simultaneous, independent measurement of temperature and strain using a tilted fibre Bragg grating
}

\author{
Edmon Chehura, Stephen W. James and Ralph P. Tatam \\ Engineering Photonics Group, School of Engineering, Cranfield University \\ Cranfield, Bedford, MK43 0AL, UK
}

\begin{abstract}
A single fibre Bragg grating is used to discriminate between temperature and strain by exploiting the core-cladding mode coupling of a tilted fibre Bragg grating (TFBG). The core and cladding modes exhibit different thermal sensivities, while the strain sensivities are approximately equal. Monitoring the core-core mode coupling resonance and the core-cladding mode coupling resonance within the spectrum of a single TFBG allows the separation of the temperature and strain induced wavelength shifts.
\end{abstract}

Keywords: tilted fibre Bragg grating, temperature and strain discrimination

\section{INTRODUCTION}

The discrimination of the temperature and strain responses of fibre Bragg grating, FBG, sensors remains an active area of research $^{1}$. The majority of the reported approaches have relied on the use of two sensors with different sensivities to temperature and strain, which has been achieved in a number of ways. Appropriate packaging can ensure that one of the sensors is shielded from one of the measurands ${ }^{2}$. FBGs with inherently different sensitivities may be used, for example, FBGs with different centre wavelengths ${ }^{3}$, FBGs written in fibres of different composition ${ }^{4}$ or with different diameters ${ }^{5}$, or FBGs fabricated under different conditions ${ }^{6,7}$. Alternatively, the measurement from an FBG may be combined with that of a different sensing technique, for example, measuring the fluorescence lifetime of ions in doped optical fibre ${ }^{8}$ or stimulated Brillouin scattering ${ }^{9}$.

Single FBG approaches have been reported. The use of the measurand induced wavelength shifts of the $1^{\text {st }}$ and $2^{\text {nd }}$ harmonics of the reflection from an FBG have been used to separate the temperature and strain responses of a single sensor element ${ }^{10}$. This technique, however, requires the use of optical sources and spectrometers operating in widely differing areas of the optical spectrum. In an extension of the technique used in [5], a single FBG may be written across a splice between fibres of different diameters ${ }^{11}$, or between fibres of different composition ${ }^{12}$. In a similar vein, techniques based on etching or packaging sections of a single FBG have been reported ${ }^{13}$. The different thermal sensitivity of the orthogonally polarised reflections from an FBG fabricated in Hi-Bi fibre has also been exploited ${ }^{14}$. In this paper the use of the properties of a single tilted fibre Bragg grating, TFBG, to facilitate the discrimination of temperature and strain is reported.

\section{PRINCIPLE}

A standard FBG consists of a refractive index modulation in the core of an optical fibre that acts to couple the fundamental forward propagating mode to the contra-propagating core mode at a wavelength that satisfies the phase matching condition. The spectrum of such a device consists of a narrow wavelength passband in reflection while the transmission spectrum exhibits a notch at the wavelength of the passband. A TFBG consists of a refractive index modulation that is purposely blazed relative to the fibre axis in order to enhance coupling between the forwardpropagating core mode and the contra-propagating cladding modes. The contra-propagating cladding modes attenuate rapidly and are therefore not observable in reflection but are observed as numerous resonances in the transmission spectrum of the TFBG. For TFBGs with small tilt angle, the forward to backward propagating mode coupling still occurs.

The exact form of the transmission spectrum is highly dependent on the tilt angle employed ${ }^{15}$. The cladding mode resonance wavelengths will be sensitive to differential changes in the indices of the cladding and core modes, which have been exploited previously to demonstrate a refractometer ${ }^{16}$. For a small tilt angle, it is possible to produce a well defined single loss band that is blue shifted with respect to the main Bragg feature ${ }^{17}$, as shown in figure 1.

Third European Workshop on Optical Fibre Sensors, Antonello Cutolo, Brian Culshaw, José Miguel López-Higuera, Eds., Proceedings of SPIE Vol. 6619, 66190I, (2007) $\cdot 0277-786$ X/07/\$18 $\cdot$ doi: 10.1117/12.738371 


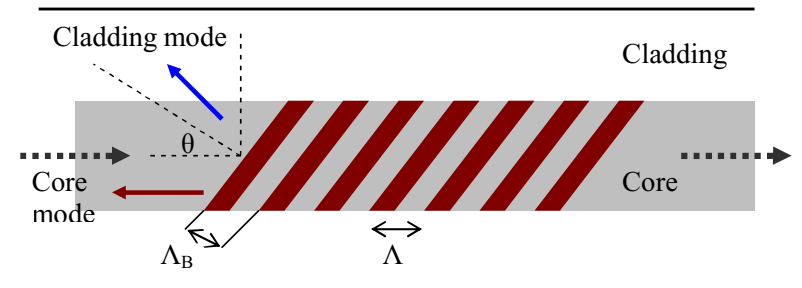

Figure 1. Schematic diagram of a TFBG. $\Lambda$; grating period, and $\theta$; blaze angle. Dashed arrows represent the forward propagating core mode.

It is known from the work carried out on another core-cladding mode coupler, the long period grating (LPG), that differences in the sensitivities of the effective indices of the core and cladding modes facilitate multi-parameter sensing using a single sensor element ${ }^{18}$. The composition and/or geometry of the fibre can be used to tailor the response of the core-cladding mode coupling wavelength to particular measurands.

The TFBG spectrum contains a spectral feature corresponding to core-core mode coupling and features corresponding to core-cladding mode coupling. The technique presented here exploits the difference in the thermal sensitivities of the core-cladding mode and core-core mode coupling resonances to facilitate simultaneous, independent measurement of strain and temperature using a single fibre grating.

\section{EXPERIMENT AND RESULTS}

A TFBG of length $5 \mathrm{~mm}$, with blaze angle $1.5^{\circ}$, was fabricated in a boron-germanium co-doped optical fibre, Fibercore PS1250, with cut off wavelength of $1213 \mathrm{~nm}$, using the near-field interference pattern of a tilted phase-mask. The photosensitivity of the fibre was enhanced by pressurizing it in hydrogen for a period of 2 weeks at a pressure of 150 bars at room temperature. The phase mask was illuminated with a UV beam at a wavelength of $244 \mathrm{~nm}$ and of average power $40 \mathrm{~mW}$. The TFBG was interrogated by coupling the output from a super luminescent diode of bandwidth $60 \mathrm{~nm}$ into the optical fibre, and monitoring the transmission using a scanning fibre Fabry-Perot interferometer of free spectral range $43 \mathrm{~nm}$ and finesse $900^{19}$. The reflection and transmission spectra are shown in figure 2.

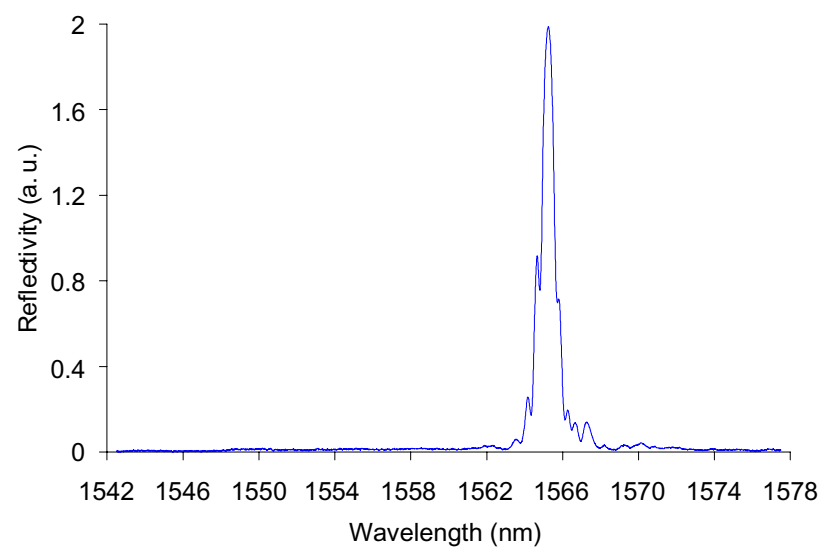

(a)

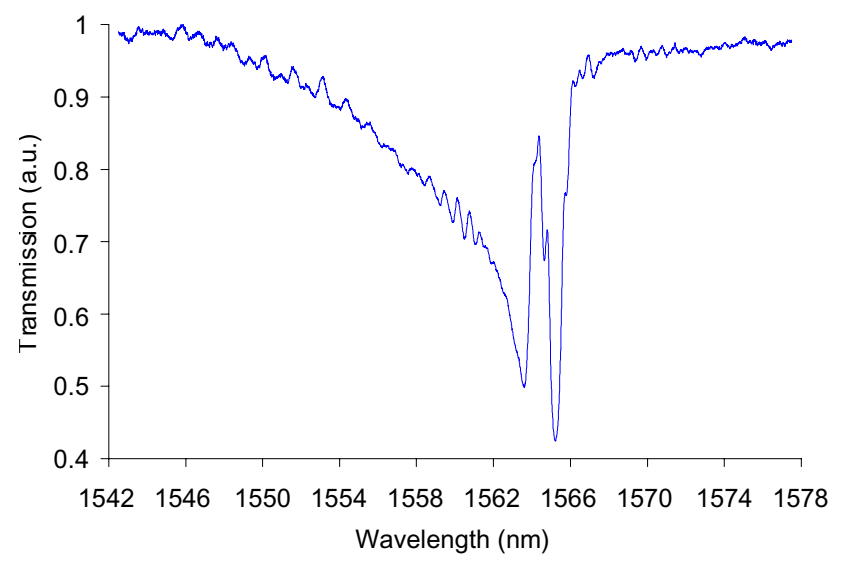

(b)

Figure 2 (a) reflection and (b) transmission spectra of a TFBG with tilt angle $1.5^{\circ}$, fabricated in hydrogen loaded boron-germanium codoped optical fibre of cut off wavelength $1213 \mathrm{~nm}$.

One side of the TFBG was anchored to a fixed block, while the other was attached to a block mounted on a translation stage, via which a known extension and thus strain could be applied. The TFBG was enclosed within a furnace that 
allowed the temperature to be controlled to within $1 \mathrm{~K}$. The strain and temperature induced wavelength shifts of the corecore, Bragg, coupling and of the core-cladding mode coupling are plotted in figure 3 . The linear fit to the data sets demonstrates that the system response can be described as linear. The contribution from any cross-sensitivity terms will not be significant over temperature ranges commonly encountered, for example, in structural monitoring ${ }^{20,21}$.

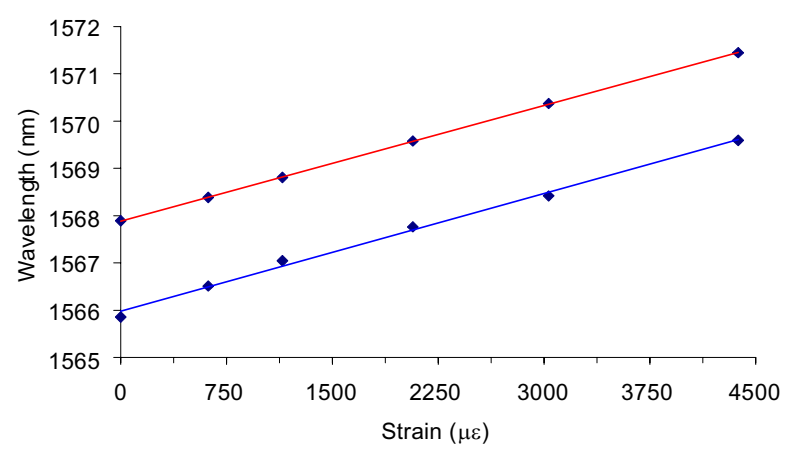

(a)

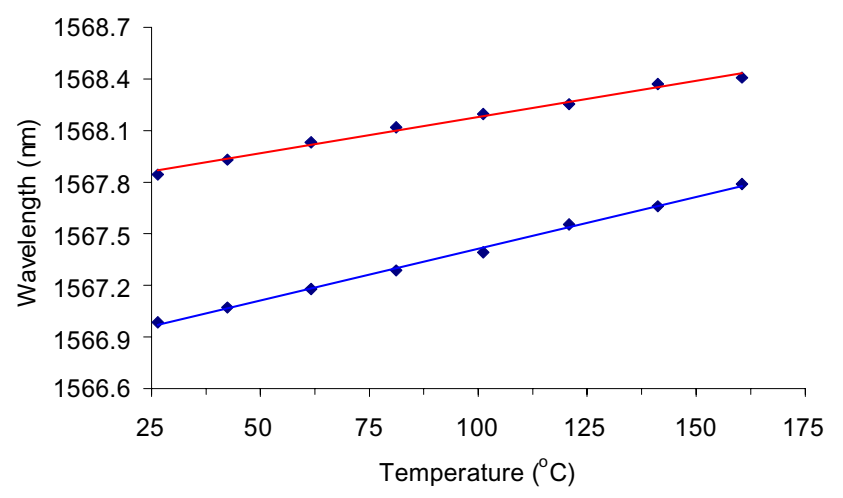

(b)

Figure 3 (a) strain and (b) temperature sensitivity of the resonance features of a TFBG with tilt angle $1.5^{\circ}$, fabricated in hydrogen loaded boron-germanium co-doped optical fibre of cut off wavelength $1213 \mathrm{~nm}$. (Red - core-core (Bragg) mode resonance and blue - core-cladding mode resonance)

The responses may be expressed in terms of a matrix, equation 1, the inverse of which would be used to determine independently temperature and strain.

$$
\left(\begin{array}{l}
\Delta \lambda_{\text {core }} \\
\Delta \lambda_{\text {clad }}
\end{array}\right)=\left(\begin{array}{ll}
K_{\text {core }, \varepsilon} & K_{\text {core }, T} \\
K_{\text {clad }, \varepsilon} & K_{\text {clad }, T}
\end{array}\right)\left(\begin{array}{c}
\varepsilon \\
T
\end{array}\right)(1)
$$

Where $\Delta \lambda_{\text {core }}$ and $\Delta \lambda_{\text {clad }}$ are the wavelength shifts of the core-core mode resonance and of the core-cladding mode resonance respectively. $\mathrm{K}_{\text {core, },}=0.816 \pm 0.003 \mathrm{pm} / \mu \varepsilon, \mathrm{K}_{\text {clad, } \varepsilon}=0.830 \pm 0.03 \mathrm{pm} / \mu \varepsilon, \mathrm{K}_{\text {core, }} \mathrm{T}=4.222 \pm 0.1 \mathrm{pm} /{ }^{\circ} \mathrm{C}$ and $\mathrm{K}_{\text {clad, }}$ ${ }_{\mathrm{T}}=6.021 \pm 0.07 \mathrm{pm} /{ }^{\circ} \mathrm{C}$ are the sensitivity coefficients for core-core mode resonance (Bragg peak) and of the corecladding mode resonance.

\section{SUMMARY}

In summary, a new approach to discriminating between the temperature and strain responses of FBG sensors has been demonstrated. The technique requires the use of a single FBG, tracking features that lie within the same spectral range, and does not require special processing or packaging of the fibre. The use of a small tilt angle that facilitates efficient coupling to a single cladding mode is beneficial, as its spectrum occupies a considerably narrower range than that 
occupied by TFBGs with larger tilt angles. Thus it allows wavelength division multiplexing to be employed. The main limitation of this approach lies in the fact that the system must be operated in transmission, which removes one of the benefits of FBG sensors - namely single ended operation.

\section{REFERENCES}

1 O Frazao, L A Ferreira, F M Araujo and J L Santos, “Applications of fibre optic grating technology to multi-parameter measurement", Fibre Int. Opt. 24, 227 - 244, 2005.

2 V V Spirin, M G Shlyagin, S V Miridonov, I Marquez, "Temperature-insensitive strain measurement using differential double Bragg grating technique", Opt. Laser Technol. 33, 43 - 46, 2001.

3 M G Xu, J L Archambault, L Reekie And J.P. Dakin, "Discrimination Between Strain And Temperature Effects Using Dual-Wavelength Fiber Grating Sensors", Electron. Lett. 30, 1085 - 1087, 1994.

4 P M Cavaleiro, F M Araujo, L A Ferreira, J L Santos, F Farahi, Simultaneous measurement of strain and temperature using Bragg gratings written in germanosilicate and boron-codoped germanosilicate fibers, IEEE Photon. Technol. Lett. 11, $1635-1637,1999$.

$5 \mathrm{~S}$ W James, M L Dockney and R P Tatam, Simultaneous independent temperature and strain measurement using infibre Bragg grating sensors, Electron. Lett. 32, 1133 - 1134, 1996.

6 X W Shu, Y Liu, D H Zhao, B Gwandu, F Floreani, L Zhang and I Bennion, Dependence of temperature and strain coefficients on fiber grating type and its application to simultaneous temperature and strain measurement, Opt. Lett. 27, $701-703,2002$.

7 X W Shu, D H Zhao, L Zhang and I Bennion, Use of dual-grating sensors formed by different types of fiber Bragg gratings for simultaneous temperature and strain measurements, Appl. Opt. 43, 2006 - 2012, 2004.

8 S A Wade, D I Forsyth, Q Guofu, X Chen, T S Chuan, W Yong, T Sun and K T V Grattan, Dual measurement of strain and temperature using the combination of Er3+-doped fibre fluorescence lifetime and a fibre Bragg grating, Meas. \& Control, 34, 175 - 178, 2001.

9 M A Davis and A D Kersey, Simultaneous measurement of temperature and strain using fibre Bragg gratings and Brillouin scattering, IEE Proc-Optoelectron. 144, 151 - 155, 1997.

10 G P Brady, K Kalli, D J Webb, D A Jackson, L Reekie and J L Archambault, Simultaneous measurement of strain and temperature using the first- and second-order diffraction wavelengths of Bragg gratings, IEE Proc -Optoelectron. 144, 156 - 161, 1997.

11 B O Guan, H Y Tam, H L W Chan, C L Choy and M S Demokan, Discrimination between strain and temperature with a single fiber Bragg grating, Microwave Opt. Tech. Lett. 33, 200 - 202, 2002.

12 O Frazao and J L Santos, Simultaneous measurement of strain and temperature using a Bragg grating structure written in germanosilicate fibres, J. Opt. A, 6, 553 - 556, 2004.

13 Z Y Zhao, S Zhang, Y S Yu, Z C Zhuo, Y Qian, Y H Cao, J Zhang, W Zheng and Y S Zhang, A simple temperatureindependent strain sensor using a fiber Bragg grating, Microwave Opt. Tech. Lett. 43, 324 - 326, 2004.

14 G H Chen, L Y Liu, H Z Jia, J M Yu, L Xu and W C Wang, Simultaneous strain and temperature measurements with fiber Bragg grating written in novel Hi-Bi optical fibre, IEEE Photon. Tech. Lett. 16, 221 - 223, 2004.

15 T Erdogan and J E Sipe, Tilted fiber phase gratings, J. Opt. Soc. Am. A, 13, 296 - 313, 1996.

$16 \mathrm{G}$ Lafont and P Ferdinand, Tilted short-period fibre-Bragg-grating-induced coupling to cladding modes for accurate refractometry, Meas. Sci. and Technol. 12, 765 - 770, 2001.

17 S Baek, Y Jeong and B Lee, Characteristics of short-period blazed fiber Bragg gratings for use as macro-bending sensors, Appl. Opt. 41, 631 - 636, 2002.

18 S W James and R P Tatam, Optical Fibre Long Period Grating Sensors: Characteristics and Application, Meas. Sci. Technol. 14, R49 - R61, 2003.

19 C-C Ye, S E Staines, S W James and R P Tatam, A polarization maintaining fibre Bragg grating interrogation system for multi-axis strain sensing, Meas. Sci. Technol. 13, 1446 - 1449, 2002.

20 G M H Flockhart, R R J Maier, J S Barton, W N MacPherson, J D C Jones, K E Chisholm, L Zhang, I Bennion, I Read, P D Foote, Quadratic behaviour of fiber Bragg grating temperature coefficients, Appl. Opt. 43 2744-2751, 2004.

21 M J O'Dwyer, C-C Ye, S W James, and R P Tatam, Thermal dependence of the strain response of optical fibre Bragg gratings Meas. Sci. Technol. 15 1607-1613, 2004. 